\title{
Performance of signal detection with trellis code for downlink non-orthogonal multiple access visible light communication
}

\author{
Dwi Astharini $^{1}$ (D) Muhamad Asvial ${ }^{1}$ (D) . Dadang Gunawan ${ }^{1}$ (D)
}

Received: 22 July 2019 / Accepted: 26 January 2021 / Published online: 16 February 2022

(c) The Author(s) 2022

\begin{abstract}
This paper examines the implementation of multi-level amplitude modulation enforced by trellis coding in a downlink nonorthogonal multiple access channel for visible light communication (VLC). The non-orthogonal transmission is applied using superposition coding and successive interference cancellation with the support of a trellis decoder. The VLC is addressed by a channel model according to Lambert. Two levels of maximum likelihood signal detection are observed and compared, each for corresponding 4 and 8 trellis-coded modulation (TCM). The power ratio allotment is examined to investigate the system performance in the bit error rate. The simulation showed that a threshold of power ratio is needed to ensure good performance of signal detection. Further simulation with successive detection had shown effective results for up to three users.
\end{abstract}

Keywords Maximum likelihood signal detection $\cdot$ Power allocation $\cdot$ VLC $\cdot$ NOMA $\cdot$ Trellis coding

\section{Introduction}

For the next decade, access point will be an ever-increasing demand, particularly with the emergence of the Internet of things, wireless sensor networks, and industry 4.0. Radio frequency access point on the other hand is limited resource under massive usage and strict regulations. Given this challenge, visible light communication (VLC) is an emerging possibility for front-end communication. It is a class of wireless optic system that uses LED instantaneously as illumination and transmission media. The transmission of lights implies license-free band and a potential of higher speed. To assist multiple mobile accesses, VLC developments have been done with wide adoptions from RF systems.

Non-orthogonal multiple access (NOMA) has reemerged in recent years in terms of enlarging the user capacity of orthogonal transmission in wireless communication [1-4]. While subcarriers are assigned in orthogonal frequency

Dadang Gunawan

guna@eng.ui.ac.id

Dwi Astharini

Dwi.astharini@ui.ac.id

Muhamad Asvial

asvial@eng.ui.ac.id

1 Department of Electrical Engineering, Universitas Indonesia, Depok, Indonesia division multiplexing (OFDM) to divide the bandwidth, NOMA was applied in allotting one carrier - or subcarrier- to cater for multiple users, by allocating different signal powers to set them apart.

In VLC, the notion of OFDM has been largely discussed, among others are [5-9]. Nevertheless, discussion on subcarrier transmission is minimal, aside from the color keying $[10,11]$. In this paper, as will be shown in the second part, i.e., the system model, NOMA is used to support multiple users accessing a single LED point. Investigations of VLC NOMA were concluded by [12-15], for on-off keying (OOK) transmission. A specific power allocation discussion for NOMA VLC was ascertained in [16] also for OOK system. As a whole, studies of VLC NOMA so far are mainly exploring the system employing OOK, along with fixed power allocation. This article is then put forward non-OOK or multi-level signaling on NOMA VLC.

Progressing to non-OOK modulation for NOMA VLC, the problem arises on the signal detection. This is instigated by the transmitter-receiver component pair in VLC, i.e., LED and photodetector, which is for the moment rather limited in comparison with those in radio transmission. On this matter, maximum likelihood signal detection (MLSD) is implemented for the successive interference cancellation. In applying it, some approaches for strategy and algorithm are examined. In [17, 18], instead of successively, joint detection was offered. In [19], ML detection was approached with 
Lagrangian, while in [20] it was approached using best channel matrix selection.

The application of a trellis decoder is a common solution offered for MLSD. This has led to convolutional coding, which has been studied on several points for VLC. It has been employed to enhance OOK-based system in [21], and in [11] trellis-coded modulation was proposed in hybrid with color keying (CSK). Trellis- or Viterbi-based coding is also found in researches on fiber optic [22-24] and free space optic [25], specifically for signal detection.

Power allocation is also an important issue, as discussed in various works. In [1] and [25] user pairing was suggested as the allocation strategy. In others, the power allocation was taken more into consideration as optimization of quite complex systems using Lagrangian in [27, 28], or Lyapunov in [29], and also with an atypical angle of network calculus in [30]. In this paper, the power allocation is approached as secondary with the performance of signal detection as the main concern.

This paper thus contributes as follows.

- We propose the implementation of TCM 4 and 8 for NOMA VLC to form a multi-level signaling transmission, to compare with OOK transmission used in previous works referred. The system proposed is aligning the modulation with signal detection simultaneously.

- The successive interference cancellation for NOMA is implemented with maximum likelihood signal detection (MLSD) enforced by trellis coding corresponding to those in point a.

- Exploration of BER performance for said system, purposely where the system performance achieved the expected standard.

- The work presented in this paper has managed to display results in which three parallel users are well catered by using TCM 4 and 8.

To the best of our knowledge, no other publication has discussed the endeavors above, especially that of point one and in conjunction with point four.

The rest of the article is arranged as follows. The second part explains the system concerned, that of VLC setting, the channel model, and NOMA transmission. The proposed system design is presented in the third part, i.e., the MLSD, continued by consideration of its application on the VLC system and the convolutional code designated. This part is completed by the signal processing model fostered for the system. The performance is then analyzed in the fourth part, followed by the conclusion.

\section{VLC system model}

Consider a downlink VLC system within one access point, which transmits to a number of users using NOMA, as pictured in Fig. 1, adapted from [14, 31, 32]. Users are depicted by their photodetectors $\mathrm{PD}$, one among $\mathrm{N}$ sharing one LED access point with $n=1,2, \ldots \mathrm{N}$. The access point allocates different power to multiple users catered.

The variable $\varphi_{1 / 2}$ is the half angle of LED, representing the effective coverage of the transmitter. The distance between the LED and PD is described as $d$, while $\theta$ is the angle of emergence, i.e., the angular position of PD with respect to the transmitter axis, and $r$ is the horizontal distance between LED and PD. Another variable is $\psi$, which shows the angle of incidence of $\mathrm{PD}$, in that $0^{\circ}$ means it is angled to face the LED and able to receive maximum power, or when faced straight up vertically, the angle formed is equivalent to $\theta$.

\subsection{Channel model}

The users receive signal transmitted through VLC channel and each has its own channel gain according to the Lambertian system model. Equations (1) through (4) express the Lambertian order of emission $\mathrm{m}$, and signal power $\mathrm{P}$, in accordance with [14, 31, 33-36].

$$
\begin{aligned}
& m=-\frac{\ln (2)}{\ln \left(\cos \left(\varphi_{\frac{1}{2}}\right)\right)} \\
& h=\frac{m+1}{2 \pi d^{2}} \cos ^{m} \theta \cdot \cos (\psi) \cdot c
\end{aligned}
$$

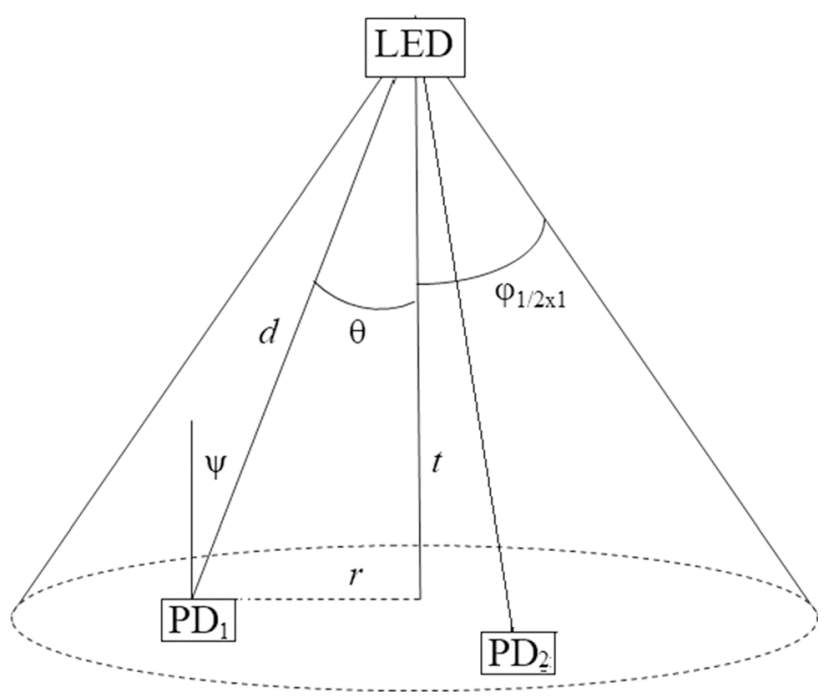

Fig. 1 VLC model 
$c=A \cdot \gamma \cdot T(\psi) \cdot g(\psi)$

$$
\begin{aligned}
P_{r} & =P_{t} \cdot h \\
& =P_{t} \frac{m+1}{2 \pi d^{2}} \cos ^{m} \theta \cdot T(\psi) \cdot g(\psi) \cdot \cos (\psi)
\end{aligned}
$$

$\varphi_{1 / 2}$ is the half angle of LED coverage; $P_{r}$ and $P_{t}$ are the transmitted and received signal power, $\mathrm{d}$ the distance of LED to $\mathrm{PD}, \theta$ is the angle of emergence between the transmitter vertical axis, $\psi$ the incidence angle expressing the reception angle of PD in reference to the vertical axis, as suggested in Fig. 2. $T$ and $g$ are filter and optical concentrator gains.

In Fig. 1, the PD's angle of incident $\psi$ appears to be of consequence from the LED's angle of emergence. However, this is not necessarily the case, as the PD's angle position is not be constrained to be perfectly vertical. The simulation within this paper is using $\psi=0$, a circumstance that allows the PD to have maximum reception from LED.

\subsection{NOMA transmission}

The NOMA concept at the transmitter is implemented as in $[12,14,31,37]$, by the use of superposition coding (SC), a summation of all parallel signals at the end of the transmitter process. For each individual signal $s_{i}$ an amount of power $P_{i}$ is allocated before the summation. At the receiver, successive interference cancellation (SIC) is applied to separate the intended signal for one user from the other. The principle of $\mathrm{SC}$ is given in Eq. (5), while Eq. (6) modeled the received power on the receiver.

$x=\sum_{i=1}^{m} x_{i}=\sum_{i=1}^{m} P_{i} s_{i}$

$y_{k}=\gamma h_{k} x+v_{k}=\gamma h_{k} \sum_{i=1}^{m} P_{i} s_{i}+v_{k}$

$x$ and $y$ are the transmitted and received signals, respectively, $m$ is the number of parallel access, $P_{i}$ is the signal power of each $i$ th parallel signal each corresponding to one access; $s$ is the data signal; $\gamma$ is the detector responsivity; $h$ is

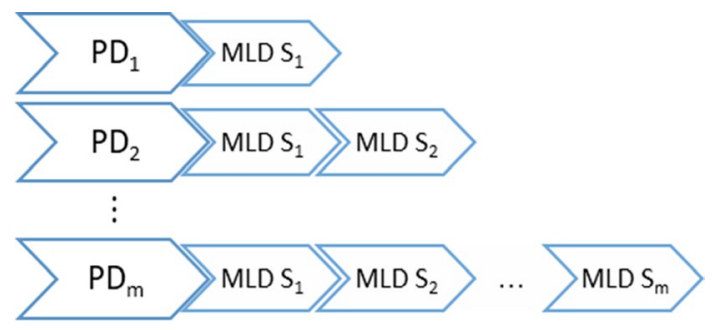

Fig. 2 Successive signal detection on the receiver the channel gain; and $\nu$ noise. The channel gain $h$ is defined by Lambertian model of Eqs. (3) and (4).

Each receiver system had pre-knowledge of their channel level in respect to other user detector, i.e., the power level it is assigned, so the separation of signals is as followed. Detector $P D_{1}$ with the highest power allocation will directly be able to determine its intended signal $s_{1}$. Detector $P D_{2}$ with lower power than that of $P D_{1}$ but higher than that $P D_{3}$, will have to determine $s_{1}$ before recognizing its intended signal $s_{2}$. Detector $P D_{3}$ goes through the process of $P D_{2}$ before successively getting $s_{3}$. And so on for further detectors, as Fig. 2 illustrates this concept.

Thus, each data signal is affected not only by transmission noise, but also by interferences from the superposition signal. The signal-to-interference and noise ratio of the NOMA signal basically can be viewed in Eq. (7), basically as in $[27,28]$

$$
\operatorname{SINR}_{i}=\frac{P_{i} s_{i} h_{i}}{\sum_{\substack{j=1 \\ j \neq i}}^{M} P_{j} s_{j} h_{j}+v}
$$

All signals intended for other users are considered interference and grouped together, along with the noise.

\section{Signal detection and encoder with trellis coding}

In this part, the proposed design is explained. Starts with the SIC concept applied as successive MLSD, followed by the trellis codes used, and completed with signal processing design for the downlink system.

\subsection{Successive signal detection}

The problem of signal detection in this system is determining how to separate the superposition signal for each user. This is accomplished by SIC in form of successive MLSD, the concept of which is depicted in Fig. 2.

Separations of signals intended for each user are done successively, i.e., decision of first signal $s_{1}$ is followed by second layer decision, which is of signal $s_{2}$, and so on up to $s_{n}$. Appointed as $P D_{1}$ would be the user with the poorest channel condition, wherein the decision has to be made only once, or one layer of signal detection. According to the fair power concept of NOMA and regarding the Lambertian channel model, this $P D_{1}$ is likely to be the user with the furthest distance or widest receiving angle from the access point LED.

For user each $U_{i}$ user, signals intended for other users are considered interference, in addition with the noise $v$. Considering the successive signal detection, as shown in 
Fig. 2, Eqs. (6)-(7) hold for the $U_{1}$ in extracting $s_{1}$. With the $s_{1}$ determined on the first layer of signal detection, the remaining signal to work on at the second layer is

$y_{2}^{\prime}=y_{2}-s_{1}=\gamma h_{2} \sum_{i=2}^{m} P_{i} s_{i}+v_{2}$

Which is generalized to be

$y_{k}^{\prime}=\gamma h_{k} \sum_{i=k}^{m} P_{i} s_{i}+v$

for the detection of $k$ th signal following previous ones. The SINR then can be rewritten as

$\operatorname{SINR}_{i}=\frac{P_{i} s_{i}}{\sum_{j=i+1}^{m} P_{j} s_{j}+v}$

For the $i$ th signal likelihood detection of NOMA VLC transmission.

\subsection{Trellis-coded modulation}

A widespread realization of MLSD is achieved using the trellis or Viterbi decoder. For more efficient decoding, a specific designed trellis can be employed for both sides of transmission, i.e., as encoder and decoder [22-24]. The TCM serves then as an amplitude modulation. As in this case, one of our objectives is to carry out non-OOK signals, and the utilization of trellis coding is suitable.

As in other constellation modulation, convolutional encoders and decoders with higher state are considerably more complex and more prone to transmission errors. In this paper, two types of convolutional encoders from [38] are investigated.

The first one is of four states, embodying a 4-PAM function. The encoder design implemented is quite a standard one, with a rate of $1 / 2$ and a constraint length $K=3$. The encoding functions employed are.

$$
\begin{aligned}
& g_{4.1}=111 \text {. } \\
& g_{4.2}=101 \text {. }
\end{aligned}
$$

For the eight-state modulation the convolutional encoder employed is with a rate of $1 / 3$ and a constraint length $K=4$. The encoding functions are.

$$
\begin{array}{llll}
g_{8.1}=1 & 0 & 0 & 0 . \\
g_{8.2}=0 & 1 & 1 & 1 . \\
g_{8.3}=1 & 1 & 0 & 1 .
\end{array} .
$$

The trellis diagram for the encoder used to ascertain fourand eight-state signaling is shown in Fig. 3.

\subsection{Design of signal process}

Various publications on optical systems have focused on the use of forward error correction practically as a
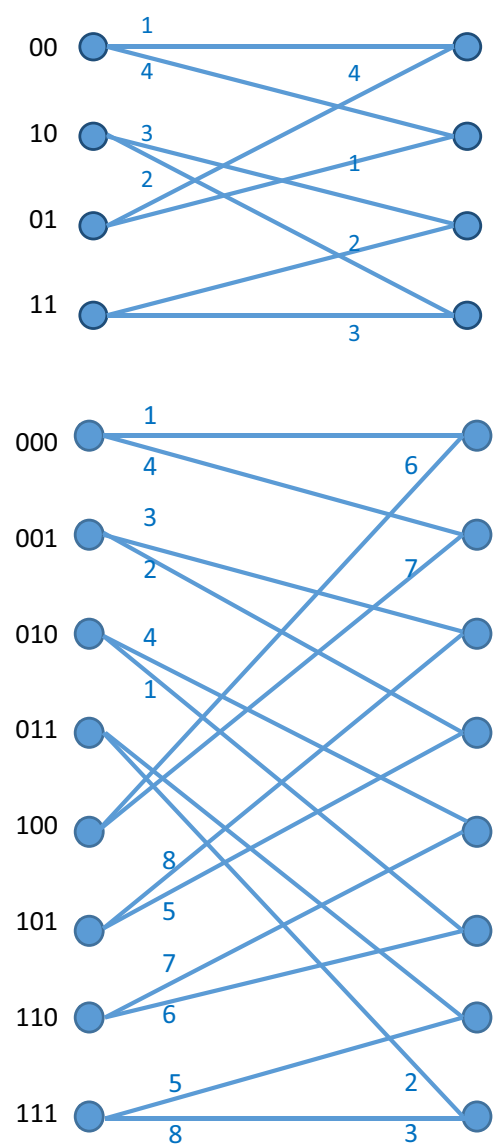

Fig. 3 Trellis diagram of a convolutional encoder applied to four-state encoder (upper) and eight-state encoder (lower)

standard or at the least highly suggested, among others are $[21,39,40]$. However, in the case proposed, we consider the trellis-coded modulation (TCM) in conjunction with the corresponding MLSD on the receiver is being able to perform the same function. In this way, the TCM serves as modulation in place of PAM, and also as encoder to minimize error on the signal detection part.

The system established is thus as shown in Fig. 4. As mentioned above, the design combined aspects of coding and modulation within one in TCM as was also suggested in [24]. One drawback is that the code rate is considerably lower than that of PAM.

As the transmitter of NOMA, amplitude modulated signals of parallel processes for multiple users are summed up to form a superposition signal according to Eq. (5). The channel is defined in the same manner as in Eqs. (1-3), so that the signal received is of Eq. (6) regarding to superpositioned signal of NOMA. Keeping in mind that the signal received along with disturbance is in the same manner as in Eq. (9), SIC is initiated as shown in Fig. 2, in form of successive maximum likelihood signal detection. 


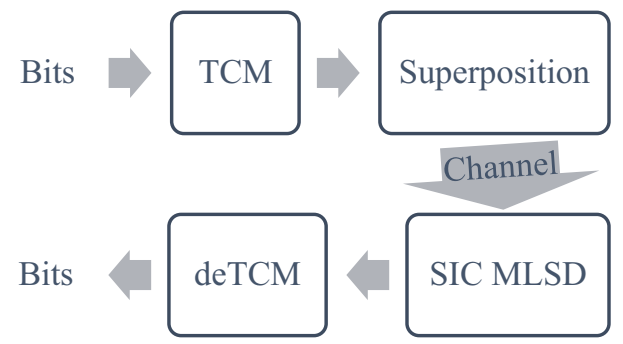

Fig. 4 System design for simulation

\section{Analytic and performance evaluation}

For the simulation, the system was implemented as shown in Fig. 4. To observe the successive signal detection for NOMA satisfactorily, simulation parameter should be considered, especially regarding the Lambertian model of VLC channel.

Performing the Lambertian model, the VLC transmission is actually represented as channel gain, or in this case, channel loss. The gain number depended on three variables: the LED coverage in $\varphi_{1 / 2}$, the relative position $d$ and angle between the LED and photodetector $\theta$, and the photodetector angle of reception $\psi$. A wide LED coverage of 90 degrees is chosen. It would transmit lower power throughout, therefore requiring stronger boost of power physically.

Acting upon that, for the following simulations, a half angle $\varphi_{1 / 2}$ of $50^{\circ}$ is chosen. As the channel gain $h$ is quite small, overall $<0.1$, this will be compensated by gains comprised in variable $c$ of Eqs. (3) and (4). The vertical distance $d$ of 2.5 was taken with the following assumption. The LED, acting as access point is lighting source of an enclosed room, situated on ceiling of $3.5 \mathrm{~m}$ height. The PDs as the user's receiver device are assumed to be located at least $1 \mathrm{~m}$ above the floor, either on tables or held by the user. The horizontal distance is denoted indirectly by the angle of emergence $\theta_{i}$. The parameter setting for performance observations is shown in Table 1.

\subsection{MLSD under varying power ratios}

The first observation is on the effectivity of the MLSD algorithm applied, in terms of the power ratio allocation. As the MLSD is a crucial part of NOMA implementation, signal power is of high concern. As stated in (5) and (6), the signal transmitted is a superpositioned of parallel processes, each with different power allocation.

The MLSD is applied successively following (9) and Fig. 2, and in each stage regarding signals for other users as interference as revealed in (7). On upfront inspection, the accomplishment of early stages interference cancellation determined the success of the following ones. Further, in order for the first stage MLSD to succeed, it has to be able
Table 1 Simulation parameters

\begin{tabular}{lll}
\hline Description & Notation & Value \\
\hline LED semi angle & $\varphi_{1 / 2}$ & $80 \mathrm{deg}$ \\
User number & $m$ & 3 \\
Angle of emergence & $\theta_{i}$ & $0,20,50 \mathrm{deg}$ \\
Incident angle & $\psi_{i}$ & 0 \\
Gains & $T \cdot g \cdot \gamma$ & 39 \\
Vertical distance & $d$ & $2.5 \mathrm{~m}$ \\
\hline
\end{tabular}

to separate $P_{1} s_{1}$ from the rest in the pile, specifically to discern all the signal constellations of $s_{1}$. With straightforward formulation P1 should be higher than.

A single signal representing one user is to be transmitted, added by a level of disturbance. The power level between the intended signal and disturbance $P S / P d$ is varied to perceive a range where the MLSD performance is reasonably better. For good measure, the SNR is set at 35, to acquire the condition when the NOMA interference would be more profound than the noise. This SNR was also considered a moderate choice in reference to [31, 32], in which OOK NOMA VLC featured good performance of low BER are achieved in similar and higher SNR, in some even on above 80. Figure 5 depicts the performance of this simulation in BER for the two levels of MLD and TCM.

From the simulation, it is seen that the detection on four TCM achieved good BER performance of below $10^{-3}$ or even $10^{-5}$, when the power ratio is above 0.8 . In our previous work it was suggested that the power is allocated according to each of user's channel condition so that $P_{i}=\alpha / h_{i}$ [29], that is inversely proportional to the channel gain. However, with the use of trellis-based MLSD, such straightforward allocation is less practical to implement. The power ratio to

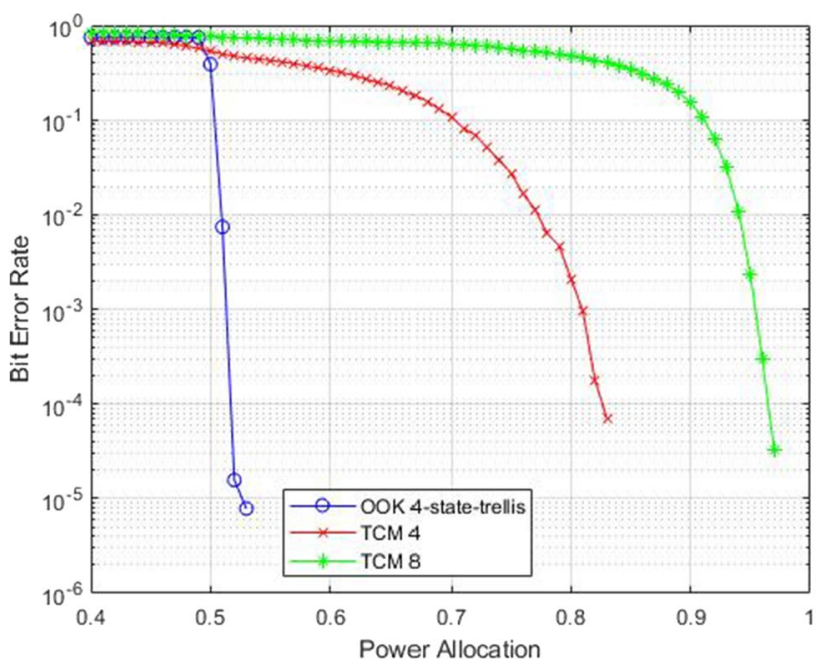

Fig. 5 MLSD performance for various power ratios on $\mathrm{SNR}=35$ 
be used for the performance observation from this point forward would thus be set to be within the boundary of $P_{i} \geq 0.8$ $P_{\text {tot. } i}$, for each $i$ th stage of MLSD employed in the successive interference cancellation. For the $8 \mathrm{TCM}$ the threshold is even higher, i.e., with minimum power ratio of 0.93 .

It remains true though, that the higher power level would be allocated for users with worse channel conditions, i.e., users with more distance or wider angle to the LED. As it was already established in the previous part that the setting used is to be of $\varphi_{1 / 2}=80^{\circ}$ with relatively similar channel gain for any $\theta$, the channel condition plays a role in determining the order of the user, in which $h_{1}<h_{2}<h_{3}$.

In successive signal detection, failure in the first stage is automatically transmitted to the next stage. The error rate of the first-tier detection is assuredly less or equal to that of the next one, as can be seen in the next part. A minimum amount of error should then be attempted, in order to ensure the next stages.

\subsection{Performance of successive MLSD for superposition signal}

Performance observation was done and to be displayed separately for $4 \mathrm{TCM}$ and $8 \mathrm{TCM}$. This is so that the observation can be made well for each user of the scheme.

For the 4 TCM, based on the previous section, the three users are assigned with power ratio of $P_{1}: P_{2}: P_{3}=85: 13.5: 1.5$. Figure 6 depicts the result graphic of BER values over SNR of $20-60$.

For the $8 \mathrm{TCM}$, the three users are assigned with power ratio of $P_{1}: P_{2}: P_{3}=93: 6.7: 0.3$. Figure 6 (lower) depicts the result graphic of BER values over SNR of 40-85. Although the power ratio of the third user is very small, with the success of the prior detection, the intended signal $s_{3}$ is still well detected, albeit under certain conditions.

With a bounded range of system parameters, the signal detection for NOMA VLC is able to detect three superposition signals with both 4 TCM and 8 TCM. However, the third user on 8 TCM could tolerate only very small level of noise on the transmission. As well to be seen, with careful choice of power allocation, three parallel users can be served soundly with both schemes of 4 and 8 states TCM.

When compared to previous publications of OOK NOMA VLC, the performance of these TCM 4 and even 8 are satisfactorily comparable. In [31, 32], the result showed that low BER were achieved on SNR higher than 100, and it was on those regions further analysis were conducted. This high SNR reflects conditions for VLC indoor transmission, especially as access points. It is necessary for the equipment to receive light signal in adequate strength and very low noise.

For comparison in Fig. 7 is shown the performance of NOMA VLC on OOK transmission, in which MLSD also employed, in this case by means of four state trellis. The
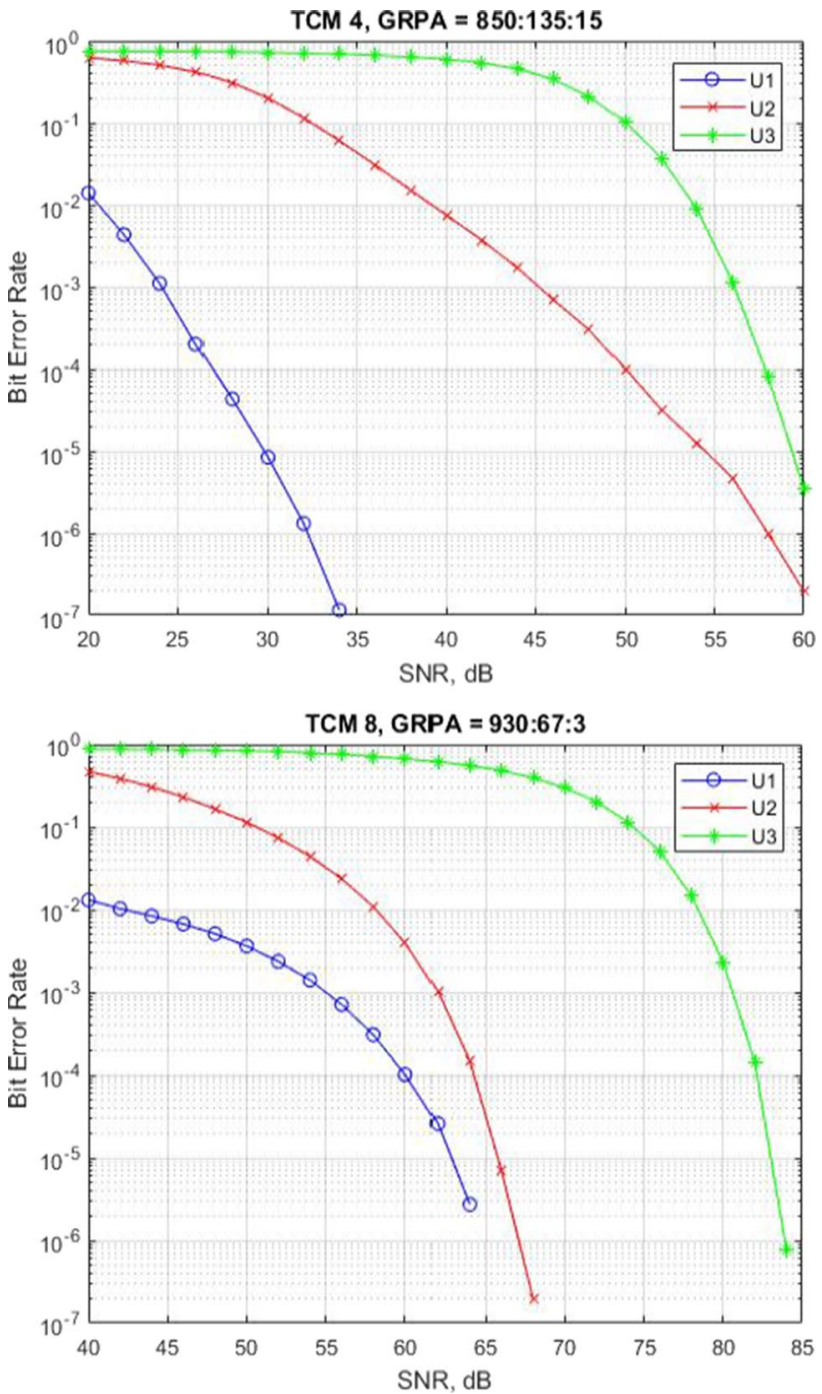

Fig. 6 Performance of three users on NOMA VLC with 4 TCM (upper) and $8 \mathrm{TCM}$ (lower)

trellis design is similar to that of upper part of Fig. 3, but the edge label would be binary signals $\{00,01,10,11\}$ of OOK transmission instead of $\{1,2,3,4\}$ of monopodal PAM signaling. With carefully assigned power ratio, all three users are able to receive good quality of signals on SNR of 30 and above.

\section{Conclusion}

In this paper, the authors proposed the application of the TCM for a NOMA VLC system, in place of a pulse amplitude one, and paired with the correspondence successive maximum likelihood signal detection. Two convolutional encoders and decoders of four- and eight-state were employed and evaluated in their performance and compared 


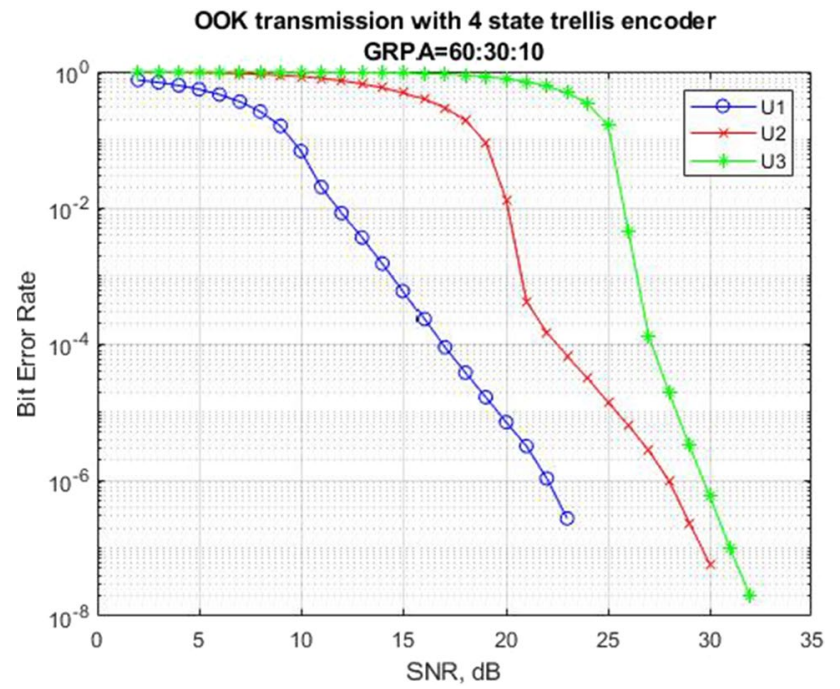

Fig. 7 Performance of three users on OOK NOMA VLC with 4 state trellis encoder MLSD

with OOK. For the successive detection to perform well, the power ratio needs to be above 0.8 on each stage on 4 TCM, and 0.93 for 8 TCM. Performance comparison for the 4 and 8 states encoders was revealed, each effectively serving three users. Considering the constraints and BER achieved, 4 TCM is more recommended for NOMA VLC.

Acknowledgements This article is part of research supported by Tadok Grant 2019 Universitas Indonesia, no. NKB-0157/UN2.R3.1/ HKP.05.00/2019.

Open Access This article is licensed under a Creative Commons Attribution 4.0 International License, which permits use, sharing, adaptation, distribution and reproduction in any medium or format, as long as you give appropriate credit to the original author(s) and the source, provide a link to the Creative Commons licence, and indicate if changes were made. The images or other third party material in this article are included in the article's Creative Commons licence, unless indicated otherwise in a credit line to the material. If material is not included in the article's Creative Commons licence and your intended use is not permitted by statutory regulation or exceeds the permitted use, you will need to obtain permission directly from the copyright holder. To view a copy of this licence, visit http://creativecommons.org/licenses/by/4.0/.

\section{References}

1. Ding, Z., Fan, P., Poor, H. V.: User pairing in non-orthogonal multiple access downlink transmissions, In: 2015 IEEE Global Communications Conference, GLOBECOM 2015 (2015)

2. Liu, F., Petrova, M.: Performance of Proportional Fair Scheduling for Downlink PD-NOMA Networks. IEEE Trans. Wirel. Commun. 17(10), 7027-7039 (2018)

3. Hsueh, W. H., Chen, Y. F.: Resource allocation for NOMA in multiuser multicarrier systems, In: 2017 International Conference on Applied System Innovation (ICASI), pp. 1626-1629 (2017)
4. Zhang, H., Yang, N., Long, K., Pan, M., Karagiannidis, G.K., Leung, V.C.M.: Secure communications in NOMA system: Subcarrier assignment and power allocation. IEEE J. Sel. Areas Commun 36(7), 1441-1452 (2018)

5. Noshad, M., Brandt-Pearce, M.: Hadamard-coded modulation for visible light communications. IEEE Trans. Commun. 64(3), 1167-1175 (2016)

6. Chen, C., Zhong, W.D., Wu, D.: Non-hermitian symmetry orthogonal frequency division multiplexing for multiple-input multipleoutput visible light communications. IEEE/OSA J. Opt. Commun. Netw. 9(1), 36-44 (2017)

7. Xu, J., Xu, W., Zhang, H., You, X.: Asymmetrically reconstructed optical OFDM for visible light communications. IEEE Photonics J. 8(1), 1-18 (2016)

8. Tejaswi, R., Narasimhan, T. L., Chockalingam, A.: Quad-LED complex modulation (QCM) for visible light wireless communication, In: 2016 IEEE Wireless Communications and Networking Conference Workshops (WCNCW), pp. 18-23 (2016).

9. Yang, C., Yang, Q., Liu, W., Li, C., Zhang, D.: Wavelet transformOFDM in indoor visible light communication, In: Optical Fiber Communication Conference, pp. W2A-62 (2015).

10. Khan, L.U.: Visible light communication: applications, architecture, standardization and research challenges. Digit. Commun. Netw. 3(2), 78-88 (2017)

11. Richard Ndjiongue, A., Ferreira, H.C.: Hybrid trellis coded modulation (HTCM) for visible light communications. IET Commun. 13(1), 85-92 (2019)

12. Guan, X., Hong, Y., Chan, C. C. K.: Non-orthogonal multiple access with multicarrier precoding in visible light communications, In: 2016 21st OptoElectronics and Communications Conference (OECC) held jointly with 2016 International Conference on Photonics in Switching (PS), pp. 1-3 (2016).

13. Yin, L., Wu, X., Haas, H.: On the performance of non-orthogonal multiple access in visible light communication, In: 2015 IEEE 26th Annual International Symposium on Personal, Indoor, and Mobile Radio Communications (PIMRC), pp. 1354-1359 (2015).

14. Marshoud, H., Kapinas, V.M., Karagiannidis, G.K., Muhaidat, S.: Non-orthogonal multiple access for visible light communications. IEEE Photonics Technol. Lett. 28(1), 51-54 (2016)

15. Kizilirmak, R. C., Rowell, C. R., Uysal, M.: Non-orthogonal multiple access (NOMA) for indoor visible light communications, In: 2015 4th International Workshop on Optical Wireless Communications (IWOW), pp. 98-101 (2015).

16. Tao, S., Yu, H., Li, Q., Tang, Y.: Performance analysis of gain ratio power allocation strategies for non-orthogonal multiple access in indoor visible light communication networks. EURASIP J. Wirel. Commun. Netw. 2018(1), 154 (2018)

17. Guan, X., Yang, Q., Chan, C.K.: Joint detection of visible light communication signals under non-orthogonal multiple access. IEEE Photonics Technol. Lett. 29(4), 377-380 (2017)

18. Sukumar, C.P., Shen, C.-A., Eltawil, A.M.: Joint detection and decoding for MIMO systems using convolutional codes: algorithm and VLSI architecture. IEEE Trans Circuits Syst. I Regul. Pap. 59(9), 1919-1931 (2012)

19. Pan, J., Ma, W.K., Jaldén, J.: MIMO detection by Lagrangian dual maximum-likelihood relaxation: reinterpreting regularized lattice decoding. IEEE Trans. Signal Process. 62(2), 511-524 (2014)

20. Jin, J.-Y., Kim, S.-C., Park, Y.-W.: Approximate ML detection with the best channel matrix selection for MIMO systems. J. Electr. Eng. Technol. 3(2), 280-284 (2010)

21. Lu, X., Li, T. J.: Exploiting RLL codes in visible light communication, In: 2016 IEEE/CIC International Conference on Communications in China (ICCC), pp. 1-6 (2016)

22. Rha, H. Y., Moon, S.-R., Kang, H.-S., Lee, S., Hwang, I., Lee, J. K.: Low complexity soft-decision Viterbi algorithm (SOVA) for 
IM/DD 56 Gbps PAM-4 system, In: IEEE Photonics Technol. Lett., pp. 1-1, (2019).

23. Ishimura, S., Kikuchi, K.: Eight-state trellis-coded optical modulation with signal constellations of four-dimensional M-ary quadrature-amplitude modulation. Opt. Express 23(5), 6692 (2015)

24. Fu, Y., Bi, M., Feng, D., Miao, X., He, H., Hu, W.: Spectral efficiency improved 2D-PAM8 trellis coded modulation for short reach optical system. IEEE Photonics J. 9(4), 1-8 (2017)

25. Song, T., Kam, P.-Y.: Efficient direct detection of M-PAM sequences with implicit CSI acquisition for the FSO system, In: 2014 IEEE Globecom Workshops (GC Wkshps), pp. 475-480 (2014)

26. Chinnadurai, S., Selvaprabhu, P., Lee, M. H.: A novel joint user pairing and dynamic power allocation scheme in MIMO-NOMA system, In: 2017 International Conference on Information and Communication Technology Convergence (ICTC), pp. 951-953 (2017).

27. Ali, M. S., Tabassum, H., Hossain, E.: Dynamic user clustering and power allocation for uplink and downlink non-orthogonal multiple access (NOMA) systems, IEEE Access, pp. 1-1, (2016).

28. Liu, F., Petrova, M.: Dynamic power allocation for downlink multi-carrier NOMA systems. IEEE Commun. Lett. 22(9), 1930$1933(2018)$

29. Bao, W., Chen, H., Li, Y., Vucetic, B.: Joint rate control and power allocation for non-orthogonal multiple access systems. IEEE J. Sel. Areas Commun. 35(12), 2798-2811 (2017)

30. Fidler, M., Rizk, A.: A guide to the stochastic network calculus. IEEE Commun. Surv. Tutorials 17(1), 92-105 (2015)

31. Yin, L., Popoola, W.O., Wu, X., Haas, H.: Performance evaluation of non-orthogonal multiple access in visible light communication. IEEE Trans. Commun. 64(12), 5162-5175 (2016)

32. Marshoud, H., Sofotasios, P.C., Muhaidat, S., Karagiannidis, G.K., Sharif, B.S.: On the performance of visible light communication systems with non-orthogonal multiple access. IEEE Trans. Wirel. Commun. 16(10), 6350-6364 (2017)

33. Marshoud, H., Sofotasios, P. C., Muhaidat, S., Karagiannidis, G. K., Sharif, B. S.: Error performance of NOMA VLC systems, In: 2017 IEEE International Conference on Communications (ICC), pp. 1-6 (2017).

34. Wu, D., et al.: Effect of optimal Lambertian order for cellular indoor optical wireless communication and positioning systems. Opt. Eng. 55(6), 066114 (2016)

35. Lin, S.-H., Liu, C., Bao, X., Wang, J.-Y.: Indoor visible light communications: performance evaluation and optimization. EURASIP J. Wirel. Commun. Netw. 2018(1), 228 (2018)

36. Astharini, D., Gunawan, D.: Discrete cosine transform and pulse amplitude modulation for visible light communication with unequally powered multiple access, In: 2018 2nd International Conference on Applied Electromagnetic Technology (AEMT), pp. 1-5 (2018).

37. Marshoud, H., Muhaidat, S., Sofotasios, P.C., Hussain, S., Imran, M.A., Sharif, B.S.: Optical non-orthogonal multiple access for visible light communication. IEEE Wirel. Commun. 25(2), 82-88 (2018)

38. Viterbi, A.: Convolutional codes and their performance in communication systems. IEEE Trans. Commun. Technol. 19(5), 751-772 (1971)

39. Alaka, S. P., Narasimhan, T. L., Chockalingam, A.: Coded index modulation for non-DC-biased OFDM in multiple LED visible light communication, In: 2016 IEEE 83rd Vehicular Technology Conference (VTC Spring), pp. 1-5 (2016).

40. Sahputra, R., Astharini, D.: Faisal: low density parity check code for OFDM transmission using bit flipping and sum product algorithm. Adv. Sci. Lett. 23(4), 3754-3757 (2017)

Publisher's Note Springer Nature remains neutral with regard to jurisdictional claims in published maps and institutional affiliations.

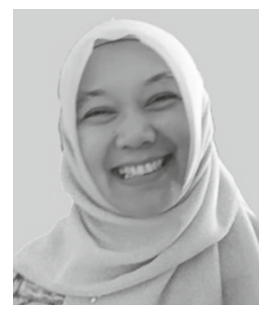

Dwi Astharini received the bachelor degree in electrical engineering (S.T) from Institut Teknologi Bandung, Indonesia, in 1997 and the M.Sc. degree in electrical engineering from Universitaet Karlsruhe, Germany, in 2001. Currently she is pursuing the Ph.D. degree in electrical engineering at Universitas Indonesia, Depok. She is also a lecturer with the Department of Electrical Engineering Universitas Al Azhar Indonesia. Her research interest include software defined telecommunication and visible light communication.

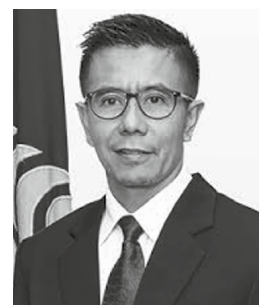

Muhamad Asvial Department of Electrical Engineering, Fakultas Teknik, Graduate Program of Telecommunications Management, Universitas Indonesia, Depok, Indonesia. Muhamad Asvial received the bachelor's degree in electrical engineering from Universitas Indonesia, in 1992, the master's degree in telecommunications engineering from Keio University, in 1998, and the Ph.D. degree from the University of Surrey, U.K., in 2003. He is currently an Associate Professor in wireless telecommunications with Universitas Indonesia, where he is also a Lecturer with the Department of Electrical Engineering and also the Vice Dean for Academic affairs with the Faculty of Engineering. His research interests include satellite communications and mobile wireless technology.

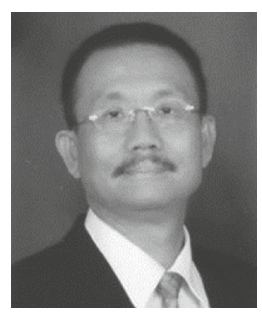

Dadang Gunawan Department of Electrical Engineering, Universitas Indonesia, Kampus Depok, Depok, Jawa Barat, Indonesia. Dadang Gunawan received the bachelor's degree in electrical engineering from the University of Indonesia in 1983, the master's degree from Keio University, Japan, in 1989, and the Ph.D. degree from the University of Tasmania, Australia, in 1995. He is currently a Professor with the Department of Electrical Engineering, Universitas Indonesia, and the Deputy Rector of Indonesian Defence University. He has published hundreds of academic papers in international journals and proceedings, as a first author or a co-author. His interests are in wireless and signal processing technology. 